\title{
Left atrial diverticula: Innocent bystanders or wolves in sheep's clothing?
}

\author{
Danny Veen BSc ${ }^{1}$ | Tobias A. Bruning MD, PhD ${ }^{2}$ | Natasja M. S. de Groot MD, PhD
}

${ }^{1}$ Department of Cardiology, Erasmus University Medical Center, Rotterdam, The Netherlands

${ }^{2}$ Department of Cardiology, Maasstad Hospital, Rotterdam, The Netherlands

\section{Correspondence}

Dr. Natasja M. S. de Groot, MD, PhD, Department of Cardiology Unit, Translational Electrophysiology, Erasmus Medical Center, Thorax Center, Room E1993,

Dr. Molewaterplein 40, PO Box 2040, 3000CA Rotterdam, The Netherlands.

Email:n.m.s.degroot@erasmusmc.n

Disclosures: None.

\begin{abstract}
Introduction: The finding of left atria diverticula (LAD) on cardiac computed tomography images obtained from patients with atrial fibrillation (AF) referred for pulmonary vein isolation is not uncommon. Prior studies reporting on LAD do not always provide definitions of LAD resulting in confusion with other anatomical structures such as left atrial accessory appendages (LAAA) and atrial aneurysms. The aim of this review is to identify an accurate definition of LAD and to describe distinctive properties between LAD and other left atrial structures, such as LAAA and aneurysms. Also, the relation between LAD and development of atrial tachyarrhythmias is discussed.
\end{abstract}

Methods: PubMed was searched for studies reporting on atrial aneurysms, left atrial diverticula, left atrial accessory appendages and atrial congenital aneurysms, resulting in 36 papers.

Results: LAD can be distinguished from LAAA by taking into account embryologic origins of the left atrium and their locations, resulting in the following definitions: (a) LAAA are contractile, trabeculated structures with circumscriptive ostia and narrow necks, originating from the primitive atria, (b) LAD are contractile, sac like structures with either smooth or trabeculated inner surfaces, circumscriptive ostia, narrow necks, and variable morphologies, originating from the embryologic common pulmonary vein, that incorporates into the LA, and (c) atrial aneurysms are non-contractile structures with wide necks and sac like bodies. There are no differences in prevalences of LAD between patients with sinus rhythm and AF.

Conclusion: The pathophysiology of LAD is not yet fully understood. It is unlikely, that LAD are related to the development of atrial tachycardia's and AF by either being a source of ectopic activity or being part of an arrhythmogenic substrate. No differences in LAD prevalences between patients with sinus rhythm and AF have been found. Thus, it is unlikely that LAD could potentially be wolves in sheep's clothing.

\section{KEYWORDS}

atrial aneurysms, atrial fibrillation, cardiac anatomy, left atrial accessory appendages, left atrial diverticula

Abbreviations: AF, atrial fibrillation; CFAE's, complex fractionated atrial electrograms; CT, computed tomography; LA, left atrium; LAA, left atrial appendage; LAAA, left atrial accessory appendages; LAD, left atria diverticula. 


\section{1 | INTRODUCTION}

The finding of left atria diverticula (LAD) on cardiac computed tomography images obtained from patients with atrial fibrillation (AF) referred for pulmonary vein isolation is not uncommon, though the exact role of LAD in the pathophysiology of AF is still unclear. ${ }^{1,2}$ Prior studies reporting on LAD do not always provide definitions of LAD resulting in confusion with other anatomical structures, such as left atrial accessory appendages (LAAA) and atrial aneurysm. There is also no uniform methodology for describing their anatomical locations. To identify locations, a method of left atrial segmenting- consisting of division of the anterior and posterior wall in four equal quadrants- was introduced. ${ }^{3}$ However, there are no anatomical boundaries used to split these four quadrants. Another methodology to describe anatomical locations uses only predefined anatomical planes, such as the ventral or dorsal plane. ${ }^{1,4-8}$ As a result, only an overall impression of regions containing these structures can be provided, but not an accurate anatomical location. This nonuniformity in methodology of localizing LAD hampers accurate labeling of atrial structures and comparison of study outcomes.

The aim of this review is to identify an accurate definition of LAD and to describe distinctive properties between LAD and other left atrial structures such as LAAA aneurysm. Also, the relation between LAD and development of atrial tachyarrhythmias is discussed

\section{1 | Definitions and properties of diverticula, aneurysms, and left atrial accessory appendages}

So far, structures extending from the cavity of the atrial wall have been ambiguously described. These structures have not only been labeled as diverticula but also as aneurysms ${ }^{9}$ or LAAA. ${ }^{10}$ However, these names are used interchangeably and accurate definitions discriminating these different types of structures are often not provided. For example, small diverticula in the left atrium were labeled as type I LAAA and large diverticula or aneurysms as type II LAAA. ${ }^{3,4,7,10}$ In the right atrium, structures extending from the endocardial cavity were not only labeled as diverticula or right atrial aneurysms but also as 'idiopathic dilatations. ${ }^{11}$ A summary of properties of diverticula, atrial aneurysms, and LAAA as described in literature is provided in the following paragraphs.

\subsection{Atrial diverticula}

Figure 1 shows an example of a typical LAD located at the left atrial posterior wall. Diverticula have been found in both the right and left atrium. They have a circumscriptive ostium and their body shows a variable morphology including cystic, sac like, tubular, and hooked shaped. Diverticula contract in synchrony with the surrounding atrial myocardium and their inner surface has been described as either smooth or trabeculated. ${ }^{3,4,6,8,10,12}$ LAD are

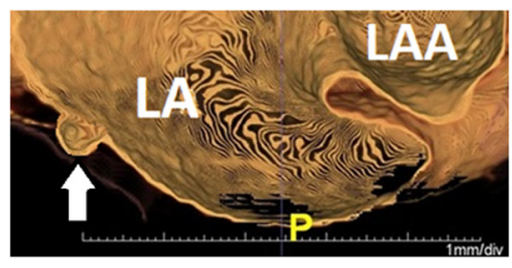

FIGURE 1 Computed tomography image from the Maasstad Hospital showing a typical example of a left atrial diverticulum on the posterior wall (white arrow). LA, left atrium; LAA, left atrial appendage; $\mathrm{P}$, posterior

either congenital or acquired origin, ${ }^{8}$ whereas right atrial diverticula are generally regarded as only congenital in origin. ${ }^{13,14}$ Sites at which congenital LAD have been observed include the LA appendage and the LA posterior wall. ${ }^{15,16}$ LA diverticula of unknown origin have been found at the anterior wall, ${ }^{8}$ anterosuperior wall, ${ }^{1,3-6}$ and the veno-atrial junction of the right superior pulmonary vein. ${ }^{17}$ In the right atrium, diverticula have been observed at the distal part of the right atrial appendage, right atrial free wall, ${ }^{18}$ within the coronary sinus, mid cardiac vein, ${ }^{13,19}$ and the right atrial posterior wall. ${ }^{14}$

\section{3 | Atrial aneurysms}

The upper panel of Figure 2 shows examples of atrial aneurysms in the right and left atrial appendages. Atrial aneurysms have been observed in both the right and left atrium and are non-contractile structures with a wide neck followed by a sac like body. (Figure 2) ${ }^{10,15,20}$ Some controversy exists on whether aneurysms are either congenital or acquired in nature. ${ }^{20}$ Atrial aneurysms were labeled as congenital in nature when located at the infero-posterior wall of the left atrium and ${ }^{20}$ the right ${ }^{21}$ or left atrial appendage (LAA). ${ }^{22}$ So far, locations of acquired aneurysms have not been reported.

\section{4 | Left atrial accessory appendages}

The lower panel of Figure 2 and Figure 3 show examples of LAAA. LAAA have been described as trabeculated or cauliflower like structures, with a circumscriptive ostium and narrow neck, contracting in synchrony with the atrial myocardium. ${ }^{4,12}$ As indicated by their name, they originate from the LAA and they are therefore regarded as congenital in nature (Figure 3). ${ }^{4,23}$ Indeed, they have been found at the antero-superior and ${ }^{6}$ lateral wall. ${ }^{3,4}$ However, in some reports atrial structures observed at sites not limited to the region of the LAA ${ }^{6}$ were also labeled as LAAA. It is questionable whether it is legitimate to label these latter structures as LAAA. One study reported ectopic activity triggering AF originating from within a LAAA located near the ostium of the right superior pulmonary vein. By ablating this focus, AF terminated. ${ }^{24}$ 
FIGURE 2 The upper panel shows examples of aneurysms (A) of the left (LAA) and right atrial appendage (RAA). (Figure is provided by courtesy of The Society of Thoracic Surgeons). In the lower panel, a computed tomography image shows an example of left atrial accessory appendages in the LAA area (white arrows). (Figure is provided by courtesy of Oxford Journals). A, aneurysm; LAA, left atrial appendage; RAA, right atrial appendage
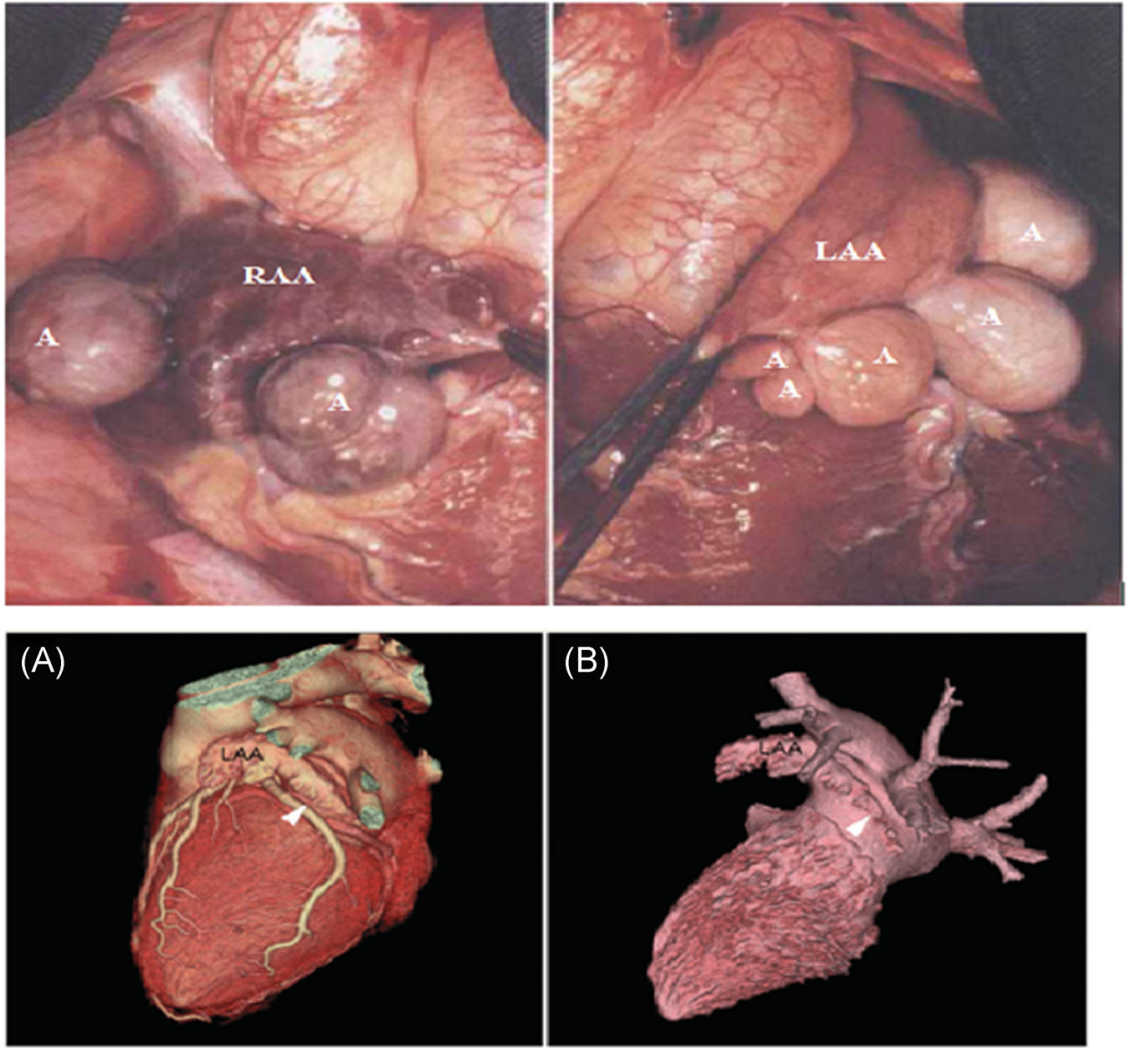

\section{5 | Discrimination between left atrial accessory appendages, diverticula, and aneurysms}

The inner surface of LAD has been described as either smooth or trabeculated, ${ }^{3,4,6,8,10,12}$ whereas LAAA have only been described as trabeculated. ${ }^{2,4,12}$ LA contractile, trabeculated structures are thus either LAD or LAAA. An approach to distinguish LAD from LAAA, is by their

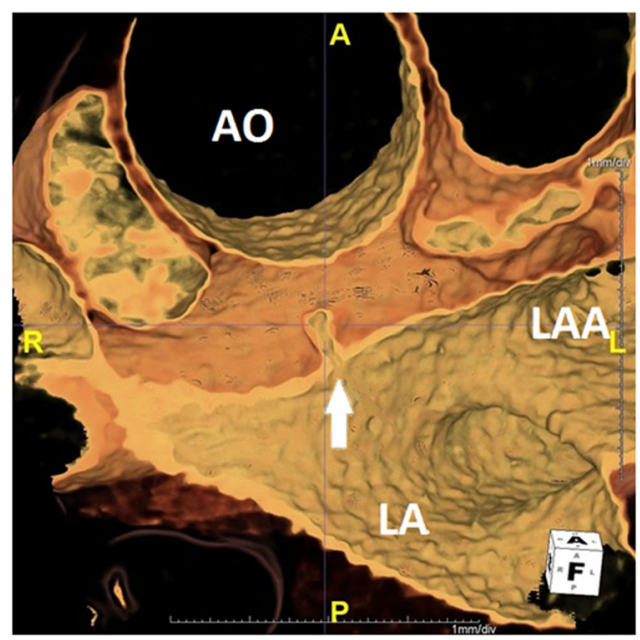

FIGURE 3 Computed tomography image from the Maasstad Hospital showing a typical example of a left atrial accessory appendage (white arrow) A, anterior; AO, aorta; L, left; LA, left atrium; LAA, left atrial appendage; $P$, posterior; $R$, right location. Taking into account the embryologic origin of the left atrium, the LAA originates from the primitive atrium, ${ }^{25}$ whereas the posterior wall of the left atrium, (depicted in purple in Figure 4) has its origin in the common pulmonary vein, that incorporates into the LA. ${ }^{27,28}$

As the LA appendage originates from the primitive atrium, only structures sharing this origin, should be labeled as LAAA (Figure 4).

As LAD and LAAA are both contractile structures, they can be easily differentiated from the non-contractile atrial aneurysms. ${ }^{10,15,20}$

Based on these observations, we propose the following definitions:

(1) LAAA are contractile, trabeculated structures with a circumscriptive ostium and a narrow neck, originating from the primitive atrium.

(2) LAD are contractile, sac like structures with either a smooth or trabeculated inner surface, a circumscriptive ostium and a narrow neck, with a variable morphology, $3,4,6,8,10,12$ originating from the embryologic common pulmonary vein, that incorporates into the LA.

(3) Atrial Aneurysms are non-contractile structures with a wide neck followed by a sac like body. ${ }^{10,15,20}$

\section{6 | Arrhythmogenic properties of diverticula}

Reports on the correlation between the presence of LAD and development of $\mathrm{AF}$ are univocal in outcome; there are no differences in prevalences of LAD between patients with sinus rhythm and AF. ${ }^{1-6}$ Thus, it is unlikely that LAD could potentially be wolves in sheep clothing. 

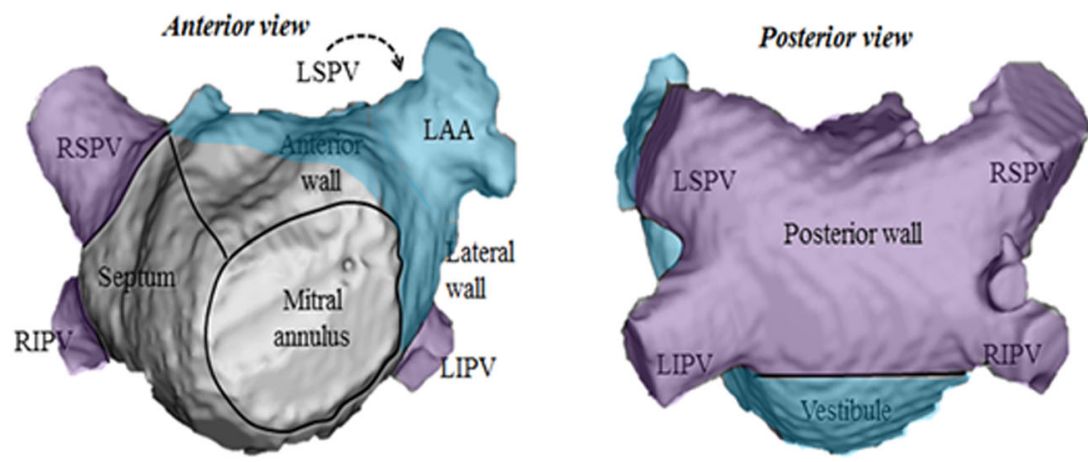

FIGURE 4 Schematic anterior and posterior view of the left atrium. The LA appendage has its origin in the primitive atrium (blue). Structures sharing the same origin as the LA appendage should only be labeled as left atrial accessory appendages. Structures located at the posterior wall, originating from the common pulmonary vein that incorporates into the left atrium, can therefore not be labeled as Left atrial accessory appendages. Image adapted and used with permission of Starreveld et al. ${ }^{26}$ LA, left atrium; LAA, LA appendage; LIPV, left inferior pulmonary vein; LSPV, left superior pulmonary vein; RIPV, right inferior pulmonary vein; RSPV, right superior pulmonary vein

Electrograms recorded from LAD can be fractionated, particularly close to its orifice. ${ }^{10,29}$ Fractionation is, for example, caused by interposition of fibrous tissue between cardiomyocytes giving rise to local asynchronous activation. ${ }^{29-31}$ Prior studies demonstrated that complex fractionated atrial electrograms (CFAE's) are indicators of the AF substrate and may serve as target sites for ablative therapy. ${ }^{30,32}$ The exact role of CFAE's recorded from LAD in the pathophysiology of $A F$ is however unknown. CFAE's are more likely to occur within a diverticulum. ${ }^{29}$ Yet, CFAE's found within diverticula are rarely target sites for ablative therapy as deliverance of radiofrequency current within a LAD may increase the likelihood of perforation of the myocardial wall by local tissue overheating. ${ }^{29,33}$

Coronary sinus diverticula may contain myocardial bundles connecting the atria and ventricles giving rise to macro-reentry tachycardia. ${ }^{34}$ Right atrial diverticula are not only associated with accessory pathways, but also with AF. For example, resection of a right atrial diverticulum in a 15 -year-old boy resulted in elimination of paroxysmal AF. ${ }^{35}$ The arrhythmogenic nature of congenital diverticula seems plausible as they most likely originate from the sinus venous which give rise to structures like the myocardium of the caval veins, the coronary sinus, crista terminalis, and the ligament of Marshall. ${ }^{25}$ All these structures are known for their role in arrhythmogenesis of tachyarrhythmias.

Also, a relation between the presence of a giant right atrial diverticulum and development of ventricular tachycardia has been described. ${ }^{36}$

\section{2 | CONCLUSION}

The pathophysiology of LAD is not yet fully understood. It is however unlikely, that the presence of LAD facilitates the development of atrial tachycardia's and AF by either being a source of ectopic activity or being part of an arrhythmogenic substrate as multiple studies, did not find differences in LAD prevalences between patients with sinus rhythm and AF. Thus, it is unlikely that LAD could potentially be wolves in sheep clothing.

\section{ACKNOWLEDGMENT}

Dr. de Groot is supported by grants from the CVON AFFIP (grant number: 914728) and VIDI (grant number: 91717339).

\section{ORCID}

Natasja M. S. de Groot (iD http://orcid.org/0000-0002-0259-6691

\section{REFERENCES}

1. Troupis J, Crossett M, Scneider-Kolsky M, Nandurkar D. Presence of accessory left atrial appendage/diverticula in a population with atrial fibrillation compared with those in sinus rhythm: a retrospective review. Int J Cardiovasc Imaging Springer Netherlands. 2012;28:375-380.

2. Patel SN, French A, Mathias H, Lyen S, Hamilton MCK, Manghat NE. Presence of left atrial diverticula, accessory appendages, and normal variant pulmonary venous anatomy diagnosed using MDCT and adverse outcomes following radiofrequency catheter ablation therapy in patients with drug-refractory atrial fibrillation: an ex. Clin Radiol. 2013;68:762-769.

3. Genç B, Solak A, Kantarci M, et al. Anatomical features and clinical importance of left atrial diverticula: MDCT findings. Clin Anat. 2014; 27:738-747.

4. Abbara S, Mundo-Sagardia JA, Hoffmann U, Cury RC. Cardiac CT assessment of left atrial accessory appendages and diverticula. AJR Am J Roentgenol. 2009;193:807-812.

5. Peng L-Q, Yu J-Q, Yang Z-G, et al. Left atrial diverticula in patients referred for radiofrequency ablation of atrial fibrillation: assessment of prevalence and morphologic characteristics by dual-source computed tomography. Circ Arrhythm Electrophysiol. 2012;5:345-350.

6. Lazoura O, Reddy T, Shriharan M, et al. Prevalence of left atrial anatomical abnormalities in patients with recurrent atrial fibrillation compared with patients in sinus rhythm using multi-slice CT. J Cardiovasc Comput Tomogr. 2012;6:268-273.

7. Duerinckx AJ, Vanovermeire $O$. Accessory appendages of the left atrium as seen during 64-slice coronary CT angiography. Int $J$ Cardiovasc Imaging. 2008;24:215-221.

8. Wan Y, He Z, Zhang L, et al. The anatomical study of left atrium diverticulum by multi-detector row CT. Surg Radiol Anat. 2009;31:191-198. 
9. Morrow AG, Behrendt DM. Congenital aneurysm (diverticulum) of the right atrium. Clinical manifestations and results of operative treatment. Circulation. 1968;38:124-128.

10. Killeen RP, Ryan R, MacErlane A, Martos R, Keane D, Dodd JD. Accessory left atrial diverticulae: contractile properties depicted with 64-slice cine-cardiac CT. Int J Cardiovasc Imaging. 2010;26:241-248.

11. Sato Y, Satokawa H, Yamamoto A, Yokoyama H, Maehara K. Giant right atrial diverticulum with thrombus formation. Asian Cardiovasc Thorac Ann. 2015;23(3):314-316.

12. Lee W-J, Chen S-J, Lin J-L, Huang Y-H, Wang T-D. Images in cardiovascular medicine. Accessory left atrial appendage: a neglected anomaly and potential cause of embolic stroke. Circulation. 2008;117: 1351-1352.

13. Binder TM, Rosenhek R, Frank H, Gwechenberger M, Maurer G, Baumgartner $\mathrm{H}$. Congenital malformations of the right atrium and the coronary sinus: an analysis based on 103 cases reported in the literature and two additional cases. Chest. 2000;117:1740-1748.

14. Petit A, Eicher JC, Louis $P$. Congenital diverticulum of the right atrium situated on the floor of the coronary sinus. Br Heart J. 1988;59:721-723.

15. Stańczyk J, Moll J, Wilczyński J. Prenatal diagnosis of a fetal left atrial diverticulum. Prenat Diagn. 1999;19:1055-1057.

16. Srinivasan V, Levinsky L, Idbeis B, Gingell RL, Pieroni DR, Subramanian S. Congenital diverticulum of the left atrium. Cardiovasc Dis. 1980;7:405-410.

17. Poh AC, Juraszek AL, Ersoy $\mathrm{H}$, et al. Endocardial irregularities of the left atrial roof as seen on coronary CT angiography. Int J Cardiovasc Imaging. 2008;24:729-734.

18. Sato Y, Satokawa H, Yamamoto A, Yokoyama H, Maehara K. Giant right atrial diverticulum with thrombus formation. Asian Cardiovasc Thorac Ann. 2015;23:314-316.

19. Wu X, Zhu R, Jiang H, Liu W. Supraventricular tachycardia and atrial flutter associated with a coronary sinus diverticulum: a case report. Exp Ther Med. 2013;5:1752-1754.

20. Morales JM, Patel SG, Jackson JH, Duff JA, Simpson JW. Left atrial aneurysm. Ann Thorac Surg. 2001;71:719-722.

21. Tejero-Hernández MÁ, Espejo-Pérez S, Suárez-de-Lezo-Cruz-Conde J. Congenital aneurysm of the right atrial appendage in a newborn: a rare anomaly. Rev Esp Cardiol. 2012;65:99-100.

22. Gold JP, Afifi HY, Ko W, Horner N, Hahn R. Congential giant aneurysms of the left atrial appendage: diagnosis and management. J Card Surg. 1996;11:147-150.

23. Lee W-J, Chen S-J, Wang T-D. Multiple accessory left atrial appendages along a semi-circular path. Eur Heart J. 2008;29:2447.

24. Killeen RP, O'Connor SA, Keane D, Dodd JD. Ectopic focus in an accessory left atrial appendage: radiofrequency ablation of refractory atrial fibrillation. Circulation. 2009;120:e60-e62.
25. Bakker ML, Christoffels VM, Moorman AFM. The cardiac pacemaker and conduction system develops from embryonic myocardium that retains its primitive phenotype. J Cardiovasc Pharmacol. 2010;56:6-15.

26. Starreveld R, LJME van der Does, NMS de Groot. Anatomical hotspots of fractionated electrograms in the left and right atrium: do they exist? Europace 2018.

27. Zhu X. Formation of the left atrium and auricle. Surgical Atlas of Cardiac Anatomy. Development of the heart chambers, 2014:127.

28. Subhadra Devi V. Cardiovascular system. Inderbir Singh's Human Embryology. Development of left atrium 11th ed., 2014:237-265.

29. Tan C, Han W, Liu X, et al. Electrophysiological characteristics of left atrial diverticulum in patients with atrial fibrillation: electrograms, impedance and clinical implications. Int J Cardiol. 2014;176:48-54.

30. Ashihara T, Haraguchi R, Nakazawa K, et al. The role of fibroblasts in complex fractionated electrograms during persistent/permanent atrial fibrillation: implications for electrogram-based catheter ablation. Circ Res. 2012;110:275-284.

31. Bakker JMT, de Wittkampf FHM. The pathophysiologic basis of fractionated and complex electrograms and the impact of tecording techniques on their detection and interpretation. Circ Arrhythmia Electrophysiol. 2010;3:204-213.

32. Nademanee K, McKenzie J, Kosar E, et al. A new approach for catheter ablation of atrial fibrillation: mapping of the electrophysiologic substrate. J Am Coll Cardiol. 2004;43:2044-2053.

33. DE Ponti R, Lumia D, Marazzi R, et al. Left atrial diverticula in patients undergoing atrial fibrillation ablation: morphologic analysis and clinical impact. J Cardiovasc Electrophysiol. 2013;24:1232-1239.

34. Hocini M, Shah AJ, Cochet H, Maury P, Denis A, Haïssaguerre M. Noninvasive electrocardiomapping facilitates previously failed ablation of right appendage diverticulum associated life-threatening accessory pathway. J Cardiovasc Electrophysiol. 2013;24:583-585.

35. Liu H, Wei L, Xia L, Wang C. An arrhythmogenic right atrial diverticulum resected through a minimally invasive approach without cardiopulmonary bypass. J Am Coll Cardiol. 2013;62:e21.

36. Hayashi T, Naito S, Kumagai K, et al. Ventricular tachycardia associated with a giant right atrial diverticulum. J Am Coll Cardiol. 2013;62:2341.

How to cite this article: Veen D, Bruning TA, de Groot NMS. Left atrial diverticula: Innocent bystanders or wolves in sheep's clothing? J Cardiovasc Electrophysiol. 2020;1-5. https://doi.org/10.1111/jce.14581 